such, though singularly altered in their general aspect; their correspondence being determined-by ascertaining their connection with other parts of the body-by tracing the series of intermediate and transitional forms which link them together, and by successive gradations lead from the most general or typical forms of organs to those, on the one hand, that are abortive and rudimentary, or to such as are exaggerated or exuberant, on the other ; and lastly-by observing the original identity of these parts in the embryo, before they have each under. gone their special modifications.

\section{STRANGULATED FEMORAL HERNIA,}

SUCCESSFUL OPERATION.

\section{To the Editor of THE LANCET.}

SIR :-Should you deem the following case worthy of publication, I shall feel honoured by seeing it inserted in your very valuable and widely-circulated Journal. I remain, Sir, your obedient servant, Charles Braddon, M.R.C.S.

Crediton, Devon, Nov. 23, 1810 .

Elizabeth Stuckey, æt. 57, an unmarried woman, and who has never given birth to a child, has been the subject of femoral hernia, on the left side, for the last twenty. five years. She attributes the formation of the tumour to a severe attack of bilious vomiting, from which she suffered extremely, about the period of the cessation of the catamenia.

Contemporaneously with the hernia, she was attacked with pain and weakness in the back, which finally issued in curvature of the spinal column; the spine being now extremely distorted, and the left shoulder very much projected. To obtain relief from the excruciating pains in the back, she very soon had recourse to opium, which she now takes in large quantities, frequently to the extent of a drachm, in the course of the day.

In addition to the foregoing affections, during the last twelve or eighteen months, the left mamma has become scirrhous, and recently ulcerated.

Such were the diseases of the poor creature, to whom I was called, on Wednesday evening, the 2sth ult. Extreme curvature of the spine, carcinomatous ulcer of the breast, the habitual practice of opium-eating, and an incarcerated hernia. On inquiry, I ascertained that the intestine had descended on Tuesday evening, and baffled all her attempts at reduction during the night. In the early part of Wednesday a surgeon was sent for, who applied the taxis, but without effect; there were no symptoms whatever of strangulation present, no sickness, no pain, no anxiety, no tension of the belly. On examination $\mathbf{I}$ found femoral hernia, rather larger than a goose egg, very hard, and manifestly filled with indurated fæces; but not at all tender to the touch, nor were the integuments swollen or inflamed. I bled her to incipient syncope, and attempted the reduction of the tumour by the taxis, but without success ; emetic tartar was administered, and nausea established, but to equally little purpose. As, however, no symptom of strangulation was present, I did not feel justified in cutting down on the intestine, but left her for the night, determining to try the effect of a tepid bath (which could not then be procured), on the following morning.

Thursday, 10 A.M. - She was placed in the warm bath till faintness and relaxation were produced, and the taxis again instituted, but without effect. Ordered to take a pill, composed of calomel, two grains, and compound extract of colocynth, eight grains, every two hours, and to have an enema with castor-oil.

8 P.M. - The enema has returned without the slightest discolouration, and the stomach rejected the medicines. No alvine evacuation since Tuesday morning. The tumour is more painful, and somewhat inflamed; the belly is also swelled, and the pulse quick; countenance extremely anxious, and very pale. There has also been vomiting. I now proposed the operation, to which the patient consented; but my seniors in the profession thought it had better be postponed till the morning, as there was no urgent symptom present.

Friday, 10 A.M.--Evidently much worse; slight hiccough has supervened, and all the other symptoms become aggravated; the operation was now decided on, which $I$ performed in the usual manner, by the inverted $\mathbf{T}$ incision. On opening the sac the intestine was found much inflamed and thickened, but only slightly congested, and the investing peritoneum was so completely softened by the inflammatory process as to peel off under the finger.* In returning the gut $I$ experienced considerable difficulty from its size, and the solidity of its contents, and was compelled to divide Poupart's ligament pretty freely, $u p$ wards and inuards. There was no omentum in the sac. She bore the operation well, and the pulse was good.

5 P.M. - There have been five alvine evacuations since the operation, without any assistance from medicine. The fæces are of dark colour, and very hard; pulse 94, soft and compressible.

11 P.M. - Two more dejections. Has been perfectly free from pain since the operation; pulse 80. To take forty minims of the liquor opii sedativus.

Saturday, 9 A.M.--Passed a quiet and tranquil night; but sleep has been prevented by * Query. Effused lymph.-ED. L. 
the constant evacuation of the bowels, there having been upwards of twelve motions during the night. Thinking this diarrhoea to proceed from inflammation of the mucous membrane of the intestine, I ordered twelve leeches immediately to the abdomen.

10 P.M.-Not so well; skin hot and dry; tongue furred; pulse hard, 85 ; belly tense. Bled to fourteen ounces, and a gruel enema to be exhibited.

Sunday, 11 A.M.-One healthy pultaceous stool in the night; countenance cheerful ; skin moist; tongue becoming clean; belly flaccid and free from pain, and not tender on pres. sure; pulse 90, soft. She expressed herself as comfortable, and much relieved by the bleeding of the preceding evening. Dressed the wound, in which adhesion had occurred throughout.

Tuesday.-Very weak, in all other respects doing well. Ordered a little meat, and a few ounces of malt liquor. From this period the recovery was rapid and permanent.

Throughout the case I was much struck with the tardy progress of the symptoms, and with the absence of that violence, which is so apt to characterise them in fenoral hernia; and this is the more remarkable, when we take into consideration the bad state of the patient's constitution, and the complication of diseases under which she laboured previously to this attack.

The tobacco enema, at all times a very questionable remedy, but more especially in cases of femoral hernia, was not here used; and the administration of it is, I believe, more frequently attended with the most dangerous (sometimes fatal) effects, than with any real advantage. It will be noticed, that during the twenty hours which immediately succeeded the operation, the bowels were moved nineteen times; in short, a complete state of diarrhoea established, though no aperient medicines had been administered. This fact, I think, manifestly points out the malpraxis of giving strong purgatives immediately after the operation; or, in other words, adding a fresh source of irritation to an already highly-irritated and inflamed membrane.

I will no farther trespass on your valuable pages, than by thus publicly expressing my grateful sense of the kind assistance of Messrs. Holman and Hainworth during the operation.

\section{HYDATIDS IN THE BRAIN.}

To the Editor of The LANCET.

Sir:-As a constant reader and subscriber to your Periodical, my attention has been drawn to your report of a discussion at the Westminster Medical Society, on cerebral disease, introduced with an assertion of the late eminent Dr. Baillie, that disorganisation of the brain is commonly found in an inverse ratio to the severity of the symptoms.

A case has immediately occurred in my practice, of the previous history and postmortem examination of which, I regret, I am not able to furnish you with a more satisfactory account.

The patient, a young man, aged nineteen years, had been working on the water here ever since he was eleven years of age; he had been exposed to every vicissitude of weather, frequently sleeping all night in an open boat, and that for many successive nights; his general health has been tolerably good, until within the last four weeks, during which he has complained of pain in his head, and has been bled in the arm twice, once by myself, and once by another surgeon here.

The young man continued working on the water until Wednesday morning, the 18th instant, when he came home, and was admitted on the same day an out-patient of the Royal Kent Dispensary. I saw him in the afternoon; the symptoms plainly showed him to be labouring under cerebral congestion ; I prescribed for him, and ordered leeches to the head; at eight in the evening I was sent for again, and on my arrival found him. dead.

I opened the head the following morning, there was considerable congestion about the scalp, and on cutting through the dura mater, I found that at the same time I had laid open a very extensive cyst, containing a vast number of lesser cysts, varying in size from that of small beads, to some which were larger than the vitreous humour of the human eye; they were perfectly spherical in form, and were filled mostly, and especially the largest, with a perfectly transparent fluid, while a few of the smaller contained a curdy cheeselike substance.

The cyst was seated in the left hemisphere of the brain, and communicated with the left ventricle, in which was a quantity of serum. The greater part of the middle and a large portion of the posterior lobe was deficient, the cyst occupying the place of the cerebral substance; compared with the opposite hemisphere, I shoulu say there was a loss of cerebral matter amounting to two. fifths.

There were no cysts in the cerebellum. I regret that $\mathrm{my}$ engagements on that day prevented my extending the examination to the other viscera.

This case is one of very extensive disorganisation of the brain, without a corresponding severity of symptoms; the patient appears to have made no complaint of his health, until within the last three or four months, and to have pursued his occupation until within thirteen hours of his decease. When the dis. ease first originated, or what period may have been occupjed in its formation, are questions I must leave to those who have more especially made pathological anatomy 